\title{
Influência da Técnica de Proporcionamento Pó/Líquido e da Fase de Inclusão nas Propriedades de uma Resina Acrílica Ativada por Micro-Ondas
} Influence of Powder/Liquid Proportioning Technique and of Pressing Stage in the Properties of an Acrylic Resin Activated by Microwaves

\author{
Juliana Rottini ${ }^{1}$, Manuela de Borges Henriques ${ }^{1}$, Fernando Freitas Portella ${ }^{1}$, Carmen Beatriz Borges Fortes ${ }^{1}$
}

\begin{abstract}
Aim: To evaluate the influence of powder/liquid proportioning method and stage of packing into a gypsum mould in Knoop microhardness, glass transition temperature, resistance to Izod impact, specific gravity and swelling degree of a microwave-cured resin. Methods: A type 5 resin was prepared using two powder/liquid proportioning methods (recommended by manufacturer and saturation technique), and packed in different stages (stricky and dough). Manufacturer stated a powder/liquid of $3: 1$. On saturation technique the liquid was dripped on powder until the complete wetting. Polymerized resins was characterized regard Knoop microhardness, glass transition temperature, resistance to Izod impact, specific gravity and swelling degree. Results: The pressing stage did not influence none of evaluated properties $(p>0.05)$, however the resins obtained by saturation technique presented Knoop microhardness, glass transition temperature, resistance to Izod impact and specific gravity higher and swelling degree lower than those obtained as stated by manufacturer $(p<0.05)$. Conclusion: Powder/liquid proportioning method influence the physical, chemical and mechanical properties of a microwavecured resin. The saturation technique produces a polymer presenting better properties in comparison to those obtained by manufacturer instructions. Pressing stage (on stricky or dough phase) did not influence none of evaluated properties.
\end{abstract}

Keywords: Acrylic resins. Dentistry. Hardness. Transition temperature.

\section{Resumo}

Objetivo: Avaliar a influência do método de proporcionamento pó/líquido e da fase de inclusão na microdureza Knoop, temperatura de transição vítrea, resistência ao impacto Izod, massa específica e grau de inchamento de uma resina acrílica ativada por micro-ondas. Materiais e Métodos: Uma resina acrílica do tipo 5 foi preparada utilizando duas formas de proporcionamento pó/líquido (proporção recomendada pelo fabricante e proporcionamento pela técnica da saturação) e incluída em diferentes fases (fase fibrilar e fase de massa). Para o proporcionamento conforme orientações do fabricante utilizou-se três partes de pó para cada parte de líquido. Na técnica da saturação o líquido foi gotejado sobre 0 pó até o completo umedecimento. As resinas polimerizadas foram caracterizadas quanto a microdureza Knoop, a temperatura de transição vítrea, a resistência ao impacto Izod, a massa específica e o grau de inchamento. Resultados: A fase de inclusão não influenciou nenhuma das propriedades avaliadas ( $p>0,05)$, contudo os grupos em que o proporcionamento foi realizado utilizando-se a técnica da saturação apresentaram microdureza Knoop, temperatura de transição vítrea, resistência ao impacto Izod e massa específica superiores, e grau de inchamento inferior em comparação com os grupos proporcionados conforme orientação do fabricante $(p<0,05)$. Conclusão: O método utilizado para o proporcionamento pó/líquido influencia as propriedades físicas, químicas e mecânicas da resina acrílica ativada por micro-ondas. A técnica de saturação produz um polímero com melhores características quando comparada ao proporcionamento recomendado pelo fabricante. O momento da inclusão (fase fibrilar ou fase de massa) não influenciou nenhuma das propriedades avaliadas.

Descritores: Dureza. Odontologia. Resinas acrílicas. Temperatura de transição.

'Laboratório de Materiais Dentários, Faculdade de Odontologia, Universidade Federal do Rio Grande do Sul, Porto Alegre, RS, Brasil.

Correspondência: Carmen Beatriz Borges Fortes

Endereço: Rua Ramiro Barcelos 2492, Porto Alegre, RS, Brasil, 90035-003 E-mail: cbbfortes@yahoo.com.br

\section{Introdução}

As resinas acrílicas são largamente utilizadas em Odontologia para a confecção de dispositivos intrabucais, para diversas finalidades, como a reabilitação de dentes perdidos ou correções ortodônticas (FRAZER et al., 2005; JAGGER et al., 1999). As próteses e demais aparatos intrabucais devem apresentar propriedades físicas, químicas e mecânicas que permitam um adequado funcionamento, higienização e confiram a durabilidade desejada (FERRACANE, 2006; RUYTER, 1982; STAFFORD et al., 1980). Destacam-se para esse fim as resinas do tipo 1, 2 e 5, sendo a polimerização dessas ativadas termicamente, quimicamente e por micro-ondas, respectivamente (ISO 20795-1: Dentistry - Base polymers). A maior vantagem da ativação por micro-ondas é a facilidade para execução do ciclo de polimerização (AZZARRI et al., 2003).

A resina acrílica ativada por micro-ondas apresenta-se na forma de um pó e um líquido, sendo o pó constituído basicamente por esferas pré-polimerizadas de polimetilmetacrilato, um iniciador (ex. peróxido de benzoila) e pigmentos; e o líquido pelo monômero de metilmetacrilato, um agente de ligação cruzada (ex. etilenoglicoldimetacrilato) e um inibidor de polimerização (ex. hidroquinona) (ANUSAVICE et al., 2013; LUNG et al., 2005). O emprego de esferas pré-polimerizadas de metilmetacrilato no pó da resina visa reduzir a contração de polimerização (ANUSAVICE et al., 2013). Dessa maneira, a contração de polimerização do metilmetacrilato puro que é de $21 \%$, passa a cerca de $7 \%$ na resina acrílica (ANUSAVICE et al., 2013). Além disso, o emprego na forma pó/líquido torna a mistura fácil e de consistência manuseável para inserção em moldes e para a confecção de próteses ou outros dispositivos intrabucais. Para isso, uma proporção de 2,5 partes de 
pó para uma parte de líquido, em peso, é utilizada. Tal proporção corresponde a 3:1, em volume, e é comumente recomendada pelos fabricantes. Porém, é possível obter uma mistura pó/líquido de viscosidade adequada misturando ambos os componentes até que não haja sobrenadante, procedimento conhecido como técnica da saturação. Após a mistura dos componentes, uma massa trabalhável é obtida, e a mistura passa por diferentes estágios.

A primeira fase da mistura é conhecida por fase arenosa, e nesse momento não há alteração em nível molecular. Em um segundo momento, denominado fase fibrilar ou pegajosa, as esferas de metilmetacrilato pré-polimerizado são atacadas pelo monômero, formando cadeias poliméricas dispersas no líquido e aumentando a viscosidade da mistura, havendo a formação de fios e apresentandose como uma mistura pegajosa. A seguir a resina entra na fase plástica ou de massa, havendo a formação de um número maior de cadeias poliméricas, mas ainda com a presença de esferas não dissolvidas, mas sem apresentar a pegajosidade da fase anterior. A partir desse momento parte do monômero evapora e a mistura entra na fase borrachóide (ANUSAVICE et al., 2013). A fase de massa é o momento em que a resina apresenta as melhores características químicas e físicas para inclusão em moldes pela técnica compressiva. O tempo para que a resina alcance a fase de massa pode variar entre as marcas comerciais, assim como também é influenciado pela proporção pó/liquido utilizada. A normatização ISO 20795-1: Dentistry - Base polymers estipula um tempo máximo de 40min para esse processo. Dessa forma, uma avaliação do efeito de diferentes métodos de proporcionamento e do momento de inclusão da mistura se faz necessária.

O objetivo desse estudo foi avaliar a influência do método de proporcionamento pó/líquido e da fase de inclusão na microdureza Knoop, temperatura de transição vítrea, resistência ao impacto Izod, massa específica e grau de inchamento de uma resina acrílica ativada por micro-ondas.

\section{Materiais e Métodos}

\section{Preparo da resina acrílica e grupos experimentais}

Uma resina acrílica do tipo 5 (Onda-Cryl, Artigos Odontológicos Clássico Ltda, São Paulo, Brasil) foi preparada utilizando-se duas formas de proporcionamento pó/líquido e incluída em diferentes fases, constituindo quatro grupos experimentais:

$\mathrm{G}_{3: 1 / \text { fibrilar: pó e liquido foram dosados conforme a orientação }}$ do fabricante, respeitando-se a proporção de três partes de pó para cada parte de líquido, medidos em volume por meio de dosador fornecido pelo fabricante. A resina foi incluída na fase fibrilar;

$\mathrm{G}_{3: 1 / \text { massa: }}$ pó e liquido foram dosados conforme a orientação do fabricante, respeitando-se a proporção de três partes de pó para cada parte de líquido, medidos em volume por meio de dosador fornecido pelo fabricante. A resina foi incluída na fase de massa;

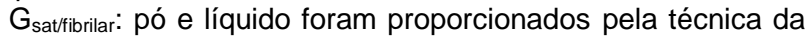
saturação, na qual o pó foi adicionado ao pote para resina e sobre esse gotejado o líquido até o completo umedecimento do pó, sem que houvesse a presença de líquido sobrenadante. Durante o gotejamento, manteve-se uma suave vibração do recipiente. A resina foi incluída na fase fibrilar.

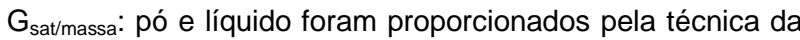
saturação, da mesma forma descrita para o grupo $\mathrm{G}_{\text {sat/fibrilar. }}$ A resina foi incluída na fase de massa.

\section{Obtenção dos corpos de prova}

Corpos de prova de resina acrílica foram obtidos a partir de matrizes retangulares de $64 \times 10 \times 3 \mathrm{~mm}$. Quatro matrizes de aço foram reproduzidas em silicone de adição (Adsil, Vigodent SA Industria e
Comércio, Rio de Janeiro, Brasil) e a impressão incluída em gesso tipo II na base de uma mufla. Após a cristalização, o gesso foi isolado com vaselina, a contra-mufla adaptada na mufla e gesso comum vertido em seu interior. Após a cristalização do gesso, o conjunto foi aberto e as matrizes metálicas removidas, sendo a área em negativo da impressão preenchida pela resina acrílica, preparada confirme descrição anterior.

A prensagem da resina acrílica foi realizada com uma carga de $1000 \mathrm{~kg}$. Após 30 minutos da prensagem, a mufla foi colocada em um forno de micro-ondas para a polimerização do material. O ciclo de polimerização constitui-se de $20 \mathrm{~min}$ com $100 \mathrm{~W}$ de potência, seguidos de $5 \mathrm{~min}$ com $400 \mathrm{~W}$. Após o resfriamento da mufla os corpos de prova foram removidos e receberam acabamento com fresas e lixas d'água de granulação 600 e 1200, por 60 segundos para cada lixa. Após foi realizado um polimento mecânico em politriz a $150000 \mathrm{rpm}$ com pedra pomes e escova de cerdas pretas por 3 min, seguido de pasta branco de espanha com escova de cerdas brancas por $3 \mathrm{~min}$

O número amostral foi definido de acordo com 0 especificado na ASTM D256: Izod Impact para o ensaio de resistência ao impacto Izod, que requer 10 corpos de prova. Para isso, o processo de obtenção dos corpos de prova foi repetido três vezes, de forma que 12 espécimes fossem obtidos para cada grupo, sendo 10 desses aleatoriamente selecionados para os ensaios. Após o ensaio de resistência ao impacto Izod, que faz a fratura dos corpos de prova em duas metades, uma das metades destinou-se ao ensaio de microdureza Knoop e de temperatura de transição vítrea, e a outra se destinou à determinação da massa específica e do grau de inchamento.

Resistência ao impacto Izod

O ensaio foi realizado de acordo com as especificações ASTM D256: Izod Impact, a exceção do entalhe no corpo de prova que não foi realizado. Os corpos de prova foram submetidos ao impacto de um pêndulo (EMIC AIC-1, São José dos Pinhais, Brasil) provocando a sua fratura. A energia de ruptura dos corpos de prova foi registrada em $\mathrm{J} / \mathrm{m}$.

\section{Microdureza Knoop (KHN)}

A microdureza Knoop dos corpos de prova foi aferida utilizando-se um microdurômetro digital (HMV 2, Shimadzu, Tóquio, Japão). Foram realizadas cinco indentações com uma carga de $25 \mathrm{~g}$ por $10 \mathrm{~s}$ em cada corpo de prova. A microdureza Knopp (KNH) foi calculada pela equação:

$$
K H N=\frac{14228 c}{d^{2}}
$$

Onde $c$ é a carga aplicada, em gramas, e $d$ é a medida da diagonal maior da indentação, em $\mu \mathrm{m}$. A microdureza Knoop de cada corpo de prova foi determinada pela média das cinco aferições.

\section{Temperatura de transição vítrea $(\mathrm{Tg})$}

A $\mathrm{Tg}$ foi determinada por calorimetria exploratória diferencial. Com o auxílio de um lâmina de bisturi, $10 \mathrm{mg}$ de resina acrílica foram removidos de cada corpo de prova, incluídas em cápsulas de alumínio e levadas a um calorímetro (DSC-4, Perkin Elmer, Beaconsfield, Inglaterra). Juntamente com cada amostra, uma cápsula de alumínio vazia foi utilizada como referência. O sistema foi submetido a dois ciclos de aquecimento, nos quais a temperatura variou de 50 a $190^{\circ} \mathrm{C}$, a uma taxa de aquecimento de $10^{\circ} \mathrm{C}$ por minuto. O gráfico fluxo de calor em função da temperatura foi obtido analisado na região correspondente ao segundo ciclo de aquecimento. A diferença de fluxo de calor entre a amostra e o sistema de referência determinou uma mudança na linha de base do gráfico, produzindo um desnível que representou a $\mathrm{Tg}$ de cada amostra. 
Massa específica $(\mu)$

A massa específica foi determinada de acordo com a especificação ASTM 792-91: Density, utilizando-se uma balança analítica de precisão (SAE 2000, Bosh, Alemanha). Acetato de $n$ butila foi utilizado como líquido de imersão para os corpos de prova para a execução do ensaio.

\section{Grau de inchamento (GI)}

Os corpos de prova tiveram sua massa aferida em uma balança analítica de precisão (SAE 2000, Bosh, Alemanha) e foram armazenados em $10 \mathrm{~mL}$ de água deionizada à $37^{\circ} \mathrm{C}$ por 7 dias. Após esse período, os corpos de prova foram armazenados secos em estufa à $37^{\circ} \mathrm{C}$ e repetidamente pesados até que atingissem estabilidade de massa.

A fração volumétrica inchada $(\Phi)$ de cada corpo de prova foi calculada pela equação:

$$
\Phi=\frac{\frac{\omega_{3}}{\mu_{3}}}{\frac{\omega_{3}}{\mu_{3}}+\frac{\omega_{2}-\omega_{3}}{\mu}}
$$

Onde $\omega_{2}$ é a massa inchada de cada corpo de prova, $\omega_{3}$ é a massa seca final do corpo de prova, $\mu_{3}$ é massa específica do corpo de prova determinada previamente e $\mu$ é a massa específica da água deionizada.

O grau de inchamento ( $\mathrm{Gl}$ ) foi calculado pelo inverso da fração volumétrica do polímero inchado, conforme a equação (FORTES, 2007):

$$
G I=\frac{1}{\Phi}
$$

\section{Análise dos dados}

Diferenças nos resultados foram verificadas pelos testes de análise de variância (ANOVA) seguida do teste de Bonferroni para dados com distribuição normal, e teste Kruskal-Wallis para dados com distribuição diferente da normal. As análises foram realizadas em um nível de significância de 5\%.

\section{Resultados}

A tabela 1 apresenta os resultados de microdureza Knoop, temperatura de transição vítrea, resistência ao impacto Izod, massa específica e grau de inchamento aferidos para os diferentes grupos experimentais. A ANOVA mostrou uma diferença significativa para os valores de microdureza Knoop $(p<0,001)$ e temperatura de transição vítrea $(p=0,009)$. Resistência ao impacto Izod $(p=0,003)$, massa específica $(p<0,001)$ e grau de inchamento $(p<0,001)$, apresentaram diferença entre os grupos, verificadas pelo teste de Kruskal-Wallis. Quando o proporcionamento pó/líquido foi realizado da mesma forma não houve diferença quanto às propriedades, contudo os grupos em que o proporcionamento foi realizado utilizando-se a técnica da saturação apresentaram microdureza Knoop, temperatura de transição vítrea, resistência ao impacto Izod e massa específica superiores, e grau de inchamento inferior em comparação com os grupos proporcionados conforme orientação do fabricante.

\section{Discussão}

A manutenção das propriedades dos aparatos intrabucais confeccionados em resina acrílica está relacionada às propriedades físicas, mecânicas e químicas das resinas (FERRACANE, 2006;
RUYTER, 1982; STAFFORD et al., 1980). Variações nos métodos de preparo das resinas acrílicas, desde o proporcionamento dos seus componentes até o polimento podem influenciar essas propriedades (ANUSAVICE et al., 2013; DOGAN et al., 1995; GEERTS et al., 2009; MOHAMED et al., 2004; SYME et al., 2001). Neste estudo, foi avaliada influencia de duas formas de proporcionamento pó/líquido e de dois momentos de inclusão, nas propriedades de uma resina acrílica polimerizada por micro-ondas. Os valores de microdureza Knoop, temperatura de transição vítrea, resistência ao impacto Izod, massa especifica e grau de inchamento aferidos estão próximos aos descritos em outros estudos (ANUSAVICE et al., 2013; FORTES, 2007; HEHN et al., 2012; MACHADO et al., 2010; TANJl et al., 2000). Verificou-se que incluir a resina acrílica na mufla na fase fibrilar ou de massa não afeta as propriedades da resina e que um proporcionamento pó/líquido realizado pela técnica da saturação produz um polímero com maior dureza, temperatura de transição vítrea, resistência ao impacto e massa específica, e menor grau de inchamento, em comparação com uma resina obtida por uma relação pó/líquido de três pra um, em volume.

Tabela 1. Média \pm desvio padrão da microdureza Knoop $(\mathrm{KNH})$, temperatura de transição vítrea $(\mathrm{Tg})$, resistência ao impacto Izod $(\mathrm{RI})$, massa específica $(\mu)$ e grau de inchamento $(\mathrm{Gl})$ de acordo com os grupos experimentais.

\begin{tabular}{cccccc}
\hline Grupo & $\mathrm{KHN}$ & $\mathrm{Tg}\left({ }^{\circ} \mathrm{C}\right)$ & $\mathrm{Rl}(\mathrm{J} / \mathrm{m})$ & $\mu\left(\mathrm{g} / \mathrm{cm}^{3}\right)$ & $\mathrm{Gl}$ \\
\hline $\mathrm{G}_{3: 1 / \text { fibrilar }}$ & $18,25 \pm 0,95^{\mathrm{b}}$ & $101,22 \pm 1,22^{\mathrm{b}}$ & $93,60 \pm 9,74^{\mathrm{b}}$ & $1,18 \pm 0,00^{\mathrm{b}}$ & $1,03 \pm 0,00^{\mathrm{a}}$ \\
$\mathrm{G}_{3: 1 / \text { massa }}$ & $17,81 \pm 0,83^{\mathrm{b}}$ & $101,13 \pm 0,99^{\mathrm{b}}$ & $94,92 \pm 12,22^{\mathrm{b}}$ & $1,18 \pm 0,00^{\mathrm{b}}$ & $1,03 \pm 0,00^{\mathrm{a}}$ \\
$\mathrm{G}_{\text {satffibrilar }}$ & $19,54 \pm 0,80^{\mathrm{a}}$ & $102,25 \pm 0,74^{\mathrm{a}}$ & $106,80 \pm 6,13^{\mathrm{a}}$ & $1,19 \pm 0,00^{\mathrm{a}}$ & $1,01 \pm 0,00^{\mathrm{b}}$ \\
$\mathrm{G}_{\text {sat/massa }}$ & $19,26 \pm 1,16^{\mathrm{a}}$ & $102,37 \pm 0,93^{\mathrm{a}}$ & $107,01 \pm 6,62^{\mathrm{a}}$ & $1,19 \pm 0,00^{\mathrm{a}}$ & $1,01 \pm 0,00^{\mathrm{b}}$ \\
\hline
\end{tabular}

As melhores propriedades alcançadas pela técnica da saturação podem ser atribuídas a menor quantidade de monômero residual e a formação de um polímero com um maior número de cadeias poliméricas e com maior peso molecular (LUNG et al., 2005). A maior massa específica e temperatura de transição vítrea, e o menor grau de inchamento apresentados pela resina obtida pela técnica da saturação suportam essa hipótese. O PMMA é um polímero termoplástico, de estrutura amorfa, que polimeriza por adição em cadeias poliméricas de comprimentos diferentes, com uma grande variação na massa molecular. O arranjo dessas cadeias não é uniforme, resultando em uma estrutura desordenada com grande energia interna. As ligações intra-moleculares são do tipo covalente, porém as ligações inter-moleculares (entre as cadeias poliméricas) são ligações fracas (do tipo van der Waals), as quais podem ser facilmente rompidas com o fornecimento de energia ao polímero. Assim, a mobilidade dessas cadeias, que é influenciada pelas condições em que a polimerização foi realizada (CANEVAROLO, 2006), pode ser caracterizada pela temperatura de transição vítrea do polímero (Tg). A Tg influencia as propriedades mecânicas e químicas do material. Alguns fatores, tais como, a massa molar (tamanho das cadeias poliméricas) e o volume livre (espaço não ocupado pelas cadeias moleculares) afetam a temperatura de transição vítrea (KAWAGUCHI et al., 2011). Cadeias menores apresentam maior mobilidade em comparação a cadeias maiores e, quanto maior 0 volume livre presente em um polímero, menor será a temperatura de transição vítrea, uma vez que maior será a facilidade das cadeias se deslocarem umas em relação às outras. Esses efeitos resultaram na maior microdureza Knoop e resistência ao impacto das resinas dos grupos $G_{\text {sat/fibrilar e }} G_{\text {sat/massa }}$ em comparação aos grupos $G_{3: 1 / \text { fibrilar }}$ e $\mathrm{G}_{3: 1 / \text { massa. }}$

Além disso, pode-se inferir que a quantidade de monômero livre também é menor na técnica da saturação. Durante a realização da técnica de saturação pó/líquido, houve uma redução na quantidade de líquido (redução aproximada de 10\%) adicionado ao pó, quando comparada à técnica recomendada pelo fabricante. Essa redução foi verificada por meio da aferição da massa de pó e líquido 
adicionados na manipulação da resina durante o preparo dos corpos de prova. A partir de um estudo piloto, constatou-se ser de difícil reprodução a quantidade em massa do pó medido utilizando o copo dosador fornecido pelo fabricante. A variabilidade da massa dosada com o copo foi de cerca de $10 \%$. Essa variação de massa promove variações na viscosidade final da mistura, além de provocar variações no tempo de trabalho e na contração de polimerização da resina (ANUSAVICE et al., 2013). O gotejamento do líquido sobre o pó, com conta-gotas graduado, permite 0 acompanhamento visual do molhamento do pó, eliminando a etapa de dosagem dos componentes e evitando a adição de monômero em excesso, o que consequentemente leva a uma redução na quantidade de monômero residual aprisionado na resina polimerizada.

Uma variação na proporção pó/líquido ideal (3 pra 1) pode influenciar a contração volumétrica de polimerização (ANUSAVICE et al., 2013). A alteração dimensional da resina acrílica não foi aferida neste estudo, porém, possivelmente o emprego da técnica da saturação promove uma redução na contração de polimerização, uma vez que um maior conteúdo de metilmetacrilato pré-polimerizado (pó) estaria sendo usado. Com essa técnica é possível o uso de uma maior quantidade de pó, mantendo-se a mistura manipulável para inserção na mulfla pela técnica compressiva, seja na fase fibrilar ou de massa.

O trabalho na fase fibrilar pode representar uma economia de tempo laboratorial, sem prejuízo às propriedades do polímero final. Provavelmente, após a inclusão na fase fibrilar, os eventos em nível molecular sigam ocorrendo no interior da mufla, não influenciando a microdureza Knoop, temperatura de transição vítrea, resistência ao impacto, massa específica e grau de inchamento, quando comparados aos polímeros obtidos a partir da inclusão da mistura na fase de massa.

A partir dos resultados obtidos é lícito inferir que os polímeros formados nos grupos $\mathrm{G}_{\text {sat/ibrilar }}$ e $\mathrm{G}_{\text {sat/massa }}$ apresentaram as seguintes características: cadeias poliméricas com massas moleculares e tamanho de cadeias maiores; volume livre entre as cadeias e/ou segmentos da cadeia menor e redução na quantidade de monômero residual. Portanto, pode-se recomendar a utilização da técnica de saturação pó/líquido, tanto para inclusão na fase fibrilar, quanto para a inclusão na fase de massa.

\section{Conclusão}

O método utilizado para o proporcionamento pó/líquido influencia a microdureza Knoop, a temperatura de transição vítrea, a resistência ao impacto Izod, a massa específica e o grau de inchamento da resina acrílica ativada por micro-ondas, sendo que a técnica de saturação produz um polímero com melhores características quando comparada ao proporcionamento recomendado pelo fabricante. Contudo, o momento da inclusão (fase fibrilar ou fase de massa) não influenciou nenhuma das propriedades avaliadas.

\section{Referências}

ASTM D256: Izod Impact. West Conshohocken, PA, USA: American Society for Testing and Materials. 1998.

ASTM 792: Density. West Conshohocken, PA, USA: American Society for Testing and Materials. 1998.

ANUSAVICE, K. J.; SHEN, C.; RAWLS, H. R. Phillips Materiais Dentários. 12. ed. Rio de Janeiro: Elsevier, 2013.
AZZARRI, M. J.; CORTIZO, M. S.; ALESSANDRINI, J. L. Effect of the curing conditions on the properties of an acrylic denture base resin microwave-polymerised. J Dent, v. 31, n. 7, p. 463-468, 2003.

CANEVAROLO S.V. Ciência dos polímeros. 3. ed. São Paulo: Artliber, 2006.

DOĞAN, A.; BEK, B.; CEVIK, N. N.; USANMAZ, A. The effect of preparation conditions of acrylic denture base materials on the level of residual monomer, mechanical properties and water absorption. $\mathbf{J}$ Dent, v. 23, n. 5, p. 313-318, Oct. 1995.

FERRACANE, J. L. Hygroscopic and hydrolytic effects in dental polymer networks. Dent Mater, v. 22, n. 3, p. 211-222, 2006.

FORTES, C. B. B. Caracterização e propriedades das resinas acrílicas de uso odontológico - Um enfoque voltado para a biossegurança. 2007. 122f. Tese (Doutorado em Ciência dos Materiais) - Programa de Pós-Graduação em Ciência dos Materiais, Universidade Federal do Rio do Sul, Porto Alegre.

FRAZER, R. Q.; BYRON, R. T.; OSBORNE, P. B.; WEST, K. P. PMMA: an essential material in medicine and dentistry. J Long Term Eff Med Implants, v. 15, n. 6, p. 629-639, 2005.

GEERTS, G. A.; DU RAND, M. The influence of powder liquid ratio on the flexural strength of fibre reinforced acrylic resin material. SADJ, $v$. 64, n. 3, p. 110, 112, 114-116, 2009.

HEHN, L.; DE MACÊDO, E. O. D.; COLLARES, F. M.; LEITUNE, V. C. B.; SAMUEL, S. M. W. Influence of peracetic acid at acrylic resin properties. Rev Odonto Cienc, v. 27, n. 3, p. 238-241, 2012.

ISO 20795-1: Dentistry - Base polymers. Geneva, Switzerland: International Organization for Standardization. 2008.

JAGGER, D. C.; HARRISON, A.; JANDT, K. D. The reinforcement of dentures. J Oral Rehabil, v. 26, n. 3, p. 185-194, 1999.

KAWAGUCHI, T.; LASSILA, L. V.; TOKUE, A.; TAKAHASHI, Y.; VALLITTU, P. K. Influence of molecular weight of polymethyl(methacrylate) beads on the properties and structure of cross-linked denture base polymer. J Mech Behav Biomed Mater, v. 4, n. 8, p. 1846-1851, 2011.

LUNG, C. Y.; DARVELL, B. W. Minimization of the inevitable residual monomer in denture base acrylic. Dent Mater, v. 21, n. 12, p. 11191128, 2005.

MACHADO, C. V.; FORTES, C. B.; COLLARES, F. M.; FORTE, M. M. C.; SAMUEL, S. M. W. Effect of different curing condition on material properties of acrylic resin for orthodontic appliances. Orthodontic Waves, v.69, n. 1, p. 18-22, Mar. 2010.

MOHAMED, S. H.; ARIFIN, A.; MOHD ISHAK, Z. A.; NIZAM, A.; SAMSUDIN, A. R. Effect of powder to liquid ratio on tensile strength and glass transition temperature of alumina filled poly methyl methacrylate (PMMA) denture base material. Med J Malaysia, v. 59, s. B, p. 147-148, 2004.

RUYTER, I. E. Methacrylate-based polymeric dental materials: conversion and related properties. Summary and review. Acta Odontol Scand, v. 40, n. 5, p. 359-376, 1982. 
STAFFORD, G. D.; BATES, J. F.; HUGGETT, R.; HANDLEY, R. W. $A$ review of the properties of some denture base polymers. J Dent, $v$. 8, n. 4, p. 292-306, 1980.

SYME, V. J.; LAMB, D. J.; LOPATTANANON, N.; ELLIS, B.; JONES, $F$. R. The effect of powder/liquid mixing ratio on the stiffness and impact strength of autopolymerising dental acrylic resins. Eur $\mathbf{J}$ Prosthodont Restor Dent, v. 9, n. 2, p. 87-91, June 2001.

TANJI, M.; DOMITTI, S. S.; CONSANI, R. L. X.; CONSANI, S.; CORRER SOBRINHO, L. Resistência ao impacto e dureza de superfície em função de diferentes tipos de resina acrílica. Salusvita, v. 19, n. 2 , p. 139-147, 2000. 